\title{
Nano-Clip based Composites with a Low Percolation Threshold and High Dielectric Constant
}

\author{
Lin Zhang, ${ }^{\mathrm{a}}$ Zhen Liu, ${ }^{\mathrm{b}}$ Xu Lu, ${ }^{\mathrm{a}}$ Guang Yang, ${ }^{\mathrm{c}}$ Xinyu Zhang, ${ }^{\mathrm{b}, *}$ and Z.-Y. Cheng ${ }^{\mathrm{a}, *}$ \\ ${ }^{a}$ Materials Research and Education Center, Auburn University, Auburn, AL 36849, USA \\ ${ }^{b}$ Department of Chemical Engineering, Auburn University, Auburn, AL 36849, USA \\ ${ }^{c}$ Electronic Materials Research Laboratory and International Center for Dielectric Research, \\ Xi'an Jiaotong University, Xi'an, 710049, China
}

*Corresponding author: chengzh@eng.auburn.edu (Z.-Y. Cheng), xzz0004@auburn.edu (Xinyu Zhang).

\begin{abstract}
An all-organic composite system using newly developed PPy nanoclips is developed. The composites have a uniform microstructure due to the unique preparation process. The composites have a very low percolation threshold $(<8 \mathrm{wt} . \%)$ and exhibit a high dielectric constant. At room temperature, the composites exhibit a dielectric constant of more than 1,000. At temperatures higher than $98{ }^{\circ} \mathrm{C}$, the composites exhibit a dielectric constant of about 2,000. More interestingly, the high dielectric constant reported here is associated with a loss much smaller than the loss reported for other CDCs using 1-D fillers. It is indicated that a new dielectric relaxation process is induced due to the mixture of PPy with P(VDF-TrFE), whose relaxation time decreases with increasing PPy content. The loss observed in the composites at low temperature including room temperature is mainly determined by this relaxation process rather than the conductivity. If this relaxation process has a strong contribution to the dielectric constant, the widely used percolation formula describing relationship between the dielectric constant and the composite cannot be used.
\end{abstract}

\section{Keywords}

Polymer, Dielectric constant, Nanocomposites, Percolation 


\section{Graphical abstracts}
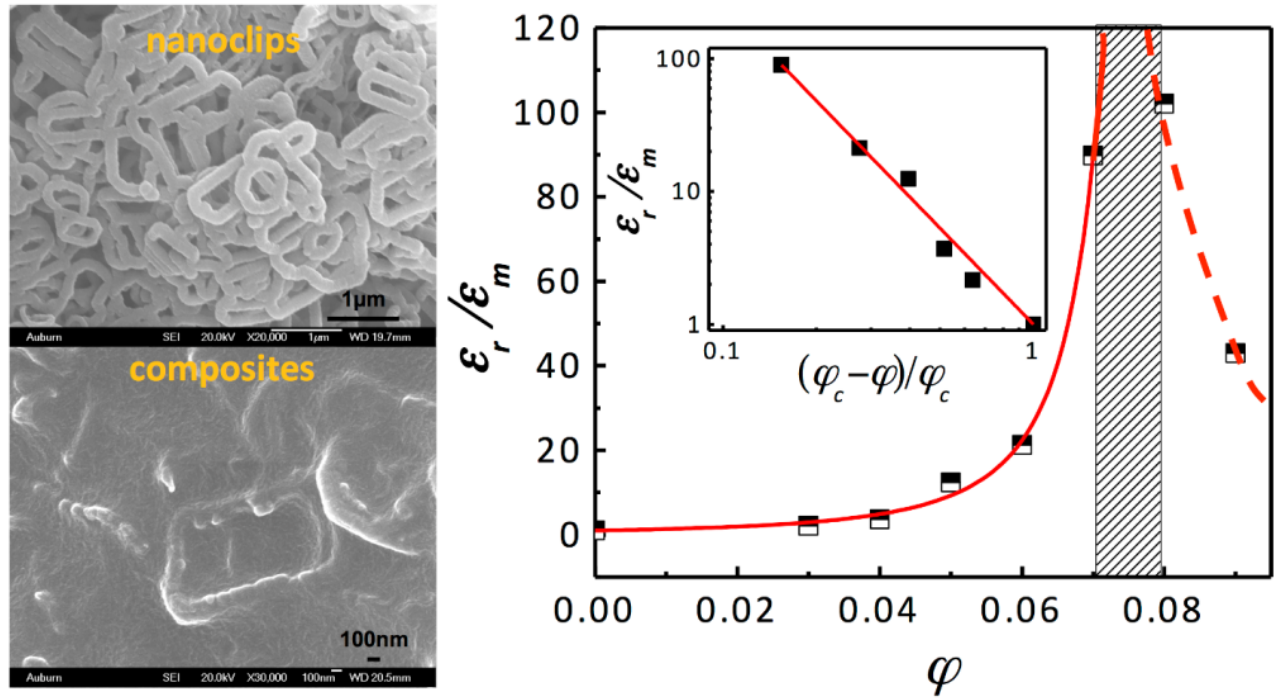


\section{Introduction}

Flexible dielectrics with a high dielectric constant $\left(\varepsilon_{r}\right)$, a low dielectric loss $(\tan \delta)$, and a low process temperature are highly desirable for many applications ranging from microelectronics, capacitors, to pulse-power and energy-storage systems [1-5]. All these properties are hard to be found in a single dielectric material. For example, dielectric polymers are flexible with a low process temperature, but their dielectric constant is low (i.e. mostly $\varepsilon_{r}<5$ ) [1], while inorganic dielectrics can exhibit a high dielectric constant, but are brittle and require a high processing temperature. The composite approach has been widely studied to improve/manipulate the materials properties. Considering all these facts, polymer-based 0-3 composites, in which a dielectric polymer used as the matrix is filled with particles of other materials, have been arousing considerable interest $[3,5]$. Based on the fillers used, these composites are classified into two categories: 1) dielectric-dielectric composites (DDCs) in which the fillers are the particles of dielectric materials with a high dielectric constant; and 2) conductor-dielectric composites (CDCs) where the fillers are particles of conducting material [3-5].

In the development of DDCs, various inorganic dielectrics with high dielectric constants $\left(10^{3} \sim 10^{4}\right)$, such as ferroelectric $\mathrm{BaTiO}_{3}[6-8], \mathrm{BaSrTiO}_{3}[9,10]$, relaxor ferroelectric $\mathrm{Pb}\left(\mathrm{Mg}_{1 / 3} \mathrm{Nb}_{2 / 3}\right) \mathrm{O}_{3}$ [11] and non-ferroelectric ceramics $\mathrm{CaCu}_{3} \mathrm{Ti}_{4} \mathrm{O}_{12}$ [12-16], have been widely studied. It is experimentally found that the DDCs barely exhibit a dielectric constant of more than 100. For most of DDCs, a high load of inorganic fillers (>50 vol.\%) is required to obtain a dielectric constant of more than 50 [5]. The high load of inorganic fillers significantly reduces the flexibility of the composites. For CDCs, the dielectric response is determined by the percolation phenomenon: when the content $(\varphi)$ of filler is lower than its percolation threshold $\left(\varphi_{c}\right)$, the composites are insulative and their dielectric constant can be written as $[4,5]$ :

$$
\varepsilon_{r} \propto\left(\varphi_{c}-\varphi\right)^{-s} \quad\left(\varphi<\varphi_{c}\right)
$$

where $s(>0)$ is a critical/power constant. This provides a unique way to develop flexible composites with a high dielectric constant if one can manipulate the $\varphi_{c}$ to a low value. CDCs 
with various conductive fillers (metal, carbon, graphite, carbon nanotubes, etc.) have been studied [3-5]. It is experimentally found that the CDCs with the $\varphi$ close to $\varphi_{c}$ can exhibit a dielectric constant of more than 1,000 [3-5].

Among the conductive fillers used so far in the development of CDCs, spherical particles of metals, such as $\mathrm{Ni}, \mathrm{Ag}, \mathrm{Cu}$, and $\mathrm{Al}$, attract much more attentions than others due to the fact that the metallic particles are inexpensive, stable, and easy to prepare [3-5]. It is found that the $\varphi_{c}$ of these composites changes over a broad range. For example, for Ni-polymer composites, the $\varphi_{c}$ varies from 17 vol. $\%$ to 57 vol.\% [17-23]. Based on physics, it was identified that the $\varphi_{c}$ should be somewhere between 33 to 75 vol. $\%$ for composites using spherical fillers if the spheres are uniformly distributed in the matrix [22, 23]. Otherwise, if the composites do not have a uniform microstructure or the filler spheres are not uniformly distributed, the $\varphi_{c}$ can be smaller [22]. However, the results would not be repeatable since the $\varphi_{c}$ would be dependent on the samples used (i.e. thickness) [23], which is undesirable for most of applications. Additionally, the interaction or adhesion between the metallic particles and polymer is weak, which reduces the mechanical performance of the composites.

As an intrinsic parameter for a composite system, the $\varphi_{c}$ is dependent on many factors, such as the shape of the filler particles. For example, when one-dimensional (1-D) fillers, like fibers, are used, a lower $\varphi_{c}$ is expected for the CDCs [4]. In fact, the CDCs with a $\varphi_{c}$ of about 10 vol.\% have been reported by using carbon fibers as a filler [24, 25]. Additionally, a better flexibility is expected for the composites using fibrous fillers than the composites using spherical fillers $[26,27]$. Both carbon fibers and carbon nanotubes have been extensively studied due to the facts that they can be easily prepared in different diameters and length/diameter ratios [28-30]. A dielectric constant of more than 1,000 has been reported in these composites with a lower $\varphi_{c}$. Unfortunately, the high dielectric constant observed in these composites is always associated with a very high loss ( $\tan \delta>10)[29,30]$. One of the reasons behind this high loss is the tangle among the fibers that results in the formation of conducting micro/nano-channels in the composites even at the $\varphi<\varphi_{c}$. The longer the length/diameter ratio is, the lower the $\varphi_{c}$ is expected [4]. However, the longer the fiber is, the 
stronger the tangle among the fiber is. This is fundamental challenge for the development CDCs using 1-D fillers.

Besides the inorganic fillers, organic fillers - conducting polymers (CPs) - have been studied, which results in all-organic composites [3-5, 31-35]. There are two main advantages by using CP fillers: 1) the electrical conductivity of the CPs can be adjusted over a broad range; and 2) a stronger interaction between the fillers and matrix can be obtained, which would make the composites with much better mechanical performance. For example, the bonding between the $\mathrm{CP}$ fillers and the polymer matrix can be easily improved using organic acids such as dodecylbenzene sulfonic acid (DBSA) [33, 35] and perfluorosulfonic acid (PFSA) [33]. The results obtained from the all-organic composites are promising. For example, in the study of PANI-P(VDF-TrFE-CTFE) all-organic composites, it is reported that the dielectric constant at $100 \mathrm{~Hz}$ of the composites can be as high as about 90 times of that of the polymer matrix [32]. Unfortunately, the development of the all-polymer composites is limited by the availability of the CP fillers. For example, the PANI fillers used in above study was particle-like, which results in a high $\varphi_{c}(\sim 26$ vol. \%) [32]. Therefore, CPs with different shape and size, especially fiber-like, would be very useful for the development of high performance all-organic composites. Recently, a unique process was developed to prepare polypyrrole (PPy) with nanofibrillar shape, i.e., 2-dimensional (2-D) PPy nanoclips [36-38].

In this paper, a high-performance CDC system using PPy 2-D nanoclips as fillers and $\mathrm{P}(\mathrm{VDF}-\mathrm{TrFE}) 70 / 30$ as the matrix is reported. These nanocomposites exhibit a very low $\varphi_{c}(<$ 8 wt.\%) and a high dielectric constant. For example, for composites with 7 and 8 wt.\% PPy at room temperature, the dielectric constants at $100 \mathrm{~Hz}$ are about 90 times and 100 times, respectively, that of the polymer matrix itself. More importantly, the high dielectric constant reported here is associated with a loss $(\sim 1)$ that is lower than the loss $\left(10^{0} \sim 10^{1}\right)$ reported in the CDCs using metallic particles and significantly lower than the loss $(>10)$ reported in other CDCs using 1-D conductive fillers. The high performance obtained in the composites reported here originates from: 1) the nanoclips used as fillers, 2) the process used in preparing 
composites. Using nanoclips as fillers inherits the advantages of using 1-D fiber fillers - the composites can have a lower $\varphi_{c}$, but prevents the formation of long conductive micro/nano-channels due to the tangle of long 1-D fibers so that the composites can exhibit a lower loss.

\section{Experimental Section}

\subsection{Synthesis of conducting PPy nanoclips}

A facile, one-step, general "oxidative template assembly" (OTA) approach was used to synthesize bulk quantities of PPy, having an unusual nanoclip-like morphology [36]. The morphology is orchestrated by an insoluble complex formed between an anionic oxidant $\left(\mathrm{S}_{2} \mathrm{O}_{8}{ }^{2-}\right)$ and a cationic surfactant. The as-produced conducting polymers possess 2-dimensional (2-D) nanostructures instead of granular structures without the templates.

In a typical experiment, $0.01 \mathrm{M}$ cetrimonium bromide $\left(\left(\mathrm{C}_{16} \mathrm{H}_{33}\right) \mathrm{N}\left(\mathrm{CH}_{3}\right)_{3} \mathrm{Br}\right)(\mathrm{CTAB})$ is dispersed in $60 \mathrm{~mL} 1 \mathrm{M} \mathrm{HCl}$ under ice bath. After being magnetically stirred 10 minutes, 0.03 M ammonium peroxydisulfate (APS) was added into the above solution and keep stirring for 10 minutes resulting in reactive template in the form of white precipitates. All solutions are cooled to $0 \sim 3{ }^{\circ} \mathrm{C}$. Pyrrole $(0.12 \mathrm{M})$ was added into the as-prepared reactive template solution, self-assembly was conducted at $0-3{ }^{\circ} \mathrm{C}$ for $24 \mathrm{hrs}$, the resulting black precipitate of PPy was suction filtered, washed with copious amounts of aq. $1 \mathrm{M} \mathrm{HCl}(3 \times 100 \mathrm{~mL})$ and acetone $(3 \times 100 \mathrm{~mL})$ and dried under freeze dry for $12 \mathrm{hrs}$. The yield of PPy nanoclips powder was $\sim 130 \mathrm{mg}$. The conductivity of PPy clips was $2.89 \mathrm{~S} / \mathrm{cm}[39]$.

\subsection{Preparation of PPy-P(VDF-TrFE) composites}

In the experiments, P(VDF-TrFE) 70/30 mol.\% copolymer from Solvey was utilized as the matrix material. The PPy-P(VDF-TrFE) composites with different PPy weight concentrations $(0,0.03,0.04,0.05,0.06,0.07,0.08$ and 0.09$)$ were prepared. The composites reported here were prepared as follows. First, P(VDF-TrFE) was dissolved in N,N dimethylformamide (DMF) with magnetic stirring for 12 hours. This was followed by the dispersion of PPy clips into the solution. They were sonicated about 30 minutes firstly, stirred for 8 hours and sonicated about 30 minutes. The copolymer solution with PPy clips was then 
casted on a quartz substrate $\left(7.6 \times 7.6 \mathrm{~cm}\right.$ from Fisher Scientific) at $70{ }^{\circ} \mathrm{C}$ for 8 hours in an oven. Finally, the as-cast film was obtained by releasing it from the quartz substrate (immersed it into D.I. water if necessary). Each as-cast film has two surfaces marked as top and bottom: the bottom is the surface contacted with the quartz substrate. The thickness of the as-cast films was about $30-40 \mu \mathrm{m}$. A hot-pressing process was then used to prepare the final composites with these as-cast films as the start materials. During hot-pressing process, four layers of the as-cast films were stacked together as a multilayer stack that was hot pressed at $150{ }^{\circ} \mathrm{C}$. The four-layer stack used in the hot-pressing process was configured in the following manner: the top of one as-cast film facing the top of the next as-cast film and the bottom of one as-cast film facing the bottom of the next as-cast film. After the hot-pressing process, the composite samples were annealed at $140{ }^{\circ} \mathrm{C}$ for $8 \mathrm{hrs}$ by placing a hot-press composite film between two glass plates.

\subsection{Characterization and measurement}

The thickness of the final composite samples was about 80 to $110 \mu \mathrm{m}$ depending on the composition. The morphology and the uniformity of the composite films were examined using JEOL JSM 7000F FE-SEM. For the characterization of the dielectric properties of the composites, the samples were sputtered with gold on both surfaces as electrodes. An Agilent 4294A impedance analyzer was used to determine the dielectric property of the samples over a frequency range from $100 \mathrm{~Hz}$ to $1 \mathrm{MHz}$ using $C p \sim \mathrm{D}$ function. In order to characterize the temperature dependence of the dielectric response, the dielectric properties of the samples were characterized at frequencies of $1 \mathrm{kHz}, 10 \mathrm{kHz}, 100 \mathrm{kHz}$ and $1 \mathrm{MHz}$ over a temperature range from $-60{ }^{\circ} \mathrm{C}$ to $140{ }^{\circ} \mathrm{C}$ using the impedance analyzer (Agilent 4294A). The heating rate was $3^{\circ} \mathrm{C} / \mathrm{min}$. The dielectric constant of the composites was calculated from the capacitance using the parallel plate mode.

\section{Results and Discussion}

As shown in Figure 1(a) and (b), each nanoclip has a length of around $1 \mu \mathrm{m}$, a width of around $0.5 \mu \mathrm{m}$, and a thickness (diameter) of around 100-200 nm. If one single nanoclip is unfolded, it would become a nanofiber with a length more than $3 \mu \mathrm{m}$ and a diameter of about 
100-200 nm. To prepare composites with a uniform microstructure, a two-step process solution cast followed by hot pressing process - was used here. This process was recently developed and its effectiveness has been demonstrated by preparing different composite systems [21, 23]. As shown in Figure 1 (c)-(f), where the composites with 5 wt.\% PPy nanoclips are presented, the composites have a uniform microstructure.

The dielectric constant and loss as well as the ac electrical conductivity $(\sigma)$ of the PPy-P(VDF-TrFE) nanocomposites with different PPy content at room temperature are shown in Figure 2, where the $\sigma$ at angular frequency $(\omega)$ was calculated using the measured dielectric constant and loss at the same angular frequency as:

$$
\sigma(\omega)=\varepsilon_{0} \cdot \omega \cdot \varepsilon_{r}^{\prime \prime}(\omega)=\varepsilon_{0} \cdot \omega \cdot \varepsilon_{r}(\omega) \cdot \tan \delta(\omega)
$$

where $\varepsilon_{r}{ }^{\prime \prime}$ is the imaginary part of the dielectric permittivity and $\varepsilon_{0}\left(=8.85 \times 10^{-12} \mathrm{~F} / \mathrm{m}\right)$ is the permittivity of free space. The dielectric constant of the composites increases with increasing filler content. A high dielectric constant $(>1,000)$ is observed in the composites with 7 wt.\% and 8 wt.\% of PPy nanoclips. The dielectric constant of the polymer matrix and nanocomposites with a low PPy content (<4 wt.\%) show a similar frequency dependence, while for composites with 4 wt.\% PPy, there is a new relaxation process arising at low frequency side, which results in a rapid increase in the loss and $\sigma$ at the low frequency. As the PPy content is increased to $5 \mathrm{wt} . \%$, this new process becomes stronger and the $\sigma$ even shows a peak-like curve at low frequency. When the PPy content is increased to $6 \mathrm{wt} . \%$, the new process results in a clear peak at a frequency of about $2 \mathrm{kHz}$ on both the loss and $\sigma$ curves, which indicates the new process is actually a dielectric relaxation process with a relaxation frequency of about $2 \mathrm{kHz}$ or a relaxation time of about $0.5 \mathrm{~ms}$. For the composites with 7 wt. $\%$ and 8 wt.\% PPy, the peak associated with the new relaxation process becomes stronger and appears at a frequency around 2 to $6 \mathrm{kHz}$. It is also observed that the frequency dependence of the dielectric constant at frequencies below the loss-peak frequency is very weakly dependent on the frequency, which confirms the new process is indeed a dielectric relaxation process. Comparing this relaxation process observed in different composites, one can conclude that its relaxation time decreases, or its relaxation frequency increases, with 
increasing PPy content. For the composites with 9 wt.\% PPy, another process arises at the low frequency side. For this composite, the $\sigma$ at low frequency is almost independent of the frequency. It is well known that the measured ac conductivity, $\sigma(\omega)$, can originate from both real conductivity $\left(\sigma_{d c}\right)$ and dielectric relaxation as:

$$
\sigma(\omega)=\sigma_{d c}+\varepsilon_{0} \varepsilon_{p}^{\prime \prime}(\omega) \cdot \omega
$$

where the $\sigma_{d c}$ is independent of the frequency, $\varepsilon_{p}^{\prime \prime}$ is the imaginary part of the real dielectric response. Therefore, the data shown in Figure 2(c) indicates that the loss or the $\sigma$ observed in all the composites except the one with 9 wt.\% PPy is mainly determined by the dielectric response rather than the electrical conduction. For the composites with 9 wt.\% PPy, a high $\sigma_{d c}$ is expected.

To further study the composition dependence, the dielectric constant at $100 \mathrm{~Hz}$ is plotted against the PPy content as shown in Figure 3(a), where the ratio of the composite's dielectric constant to the matrix's dielectric constant $\left(\varepsilon_{m}\right)$ is used. As described by Eq. (1), the dielectric constant of the CDCs increases with increasing $\varphi$ when $\varphi<\varphi_{c}$. However, if the $\varphi>\varphi_{c}$, the composites are conductive, where $\varepsilon_{r}$ decreases with increasing $\varphi$. Therefore, the data shown in Figure 3(a) indicates that the $\varphi_{c}$ should be somewhere between 7 to $8 \mathrm{wt} . \%$ (dark area) for the composites reported here. That is, the composites with 7 wt.\% PPy should be in the dielectric regime, while the composites with 8 wt.\% PPy should be in the conductive regime. Therefore, all-organic composites reported here have a very low $\varphi_{c}$.

To fit the experimental results, Equation (1) is usually written as [4, 5]:

$$
\varepsilon_{r}=\varepsilon_{m}\left(\frac{\varphi_{c}-\varphi}{\varphi_{c}}\right)^{-s} \quad \text { or } \quad \frac{\varepsilon_{r}}{\varepsilon_{m}}=\left(\frac{\varphi_{c}-\varphi}{\varphi_{c}}\right)^{-s} \quad\left(\varphi<\varphi_{c}\right)
$$

Equation (1)/(4) was originally introduced for a random binary system [40], but is widely used to analyze the results of 0-3 composites [3-5]. When Eq. (4) is used to fit the experimental data shown in Figure 3(a), it is obtained that the $\varphi_{c}$ is $8.3 \mathrm{wt} . \%$ and the $s$ is 2.4 . That is, the fitting constant $\varphi_{c}$ is more than 8 wt. $\%$, which contradicts with the results shown in Figure 3 (a). Regarding the value of the fitting constant $s$, the numerical simulation 
suggested that $s \approx 0.7[4,5]$. However, based on the experimental results, it is reported that the $s$ changes over a broad range: as low as 0.167 [41], and as high as 1.78 [42]. In other words, the $s$ value obtained here is considerable higher than other reported results.

To determine the physics behind the contradiction between the experimental data shown in Figure 3(a) and the fitting constant $\varphi_{c}$ and to find the reason for the abnormal high value of fitting constant $s$, Equation (4) is used to fit the dielectric constant obtained in the composites at different frequencies. It is found that both the fitting constant $\varphi_{c}$ and $s$ change with the frequency selected/used as shown in Figure 3(b). For example, using the dielectric constant at $1 \mathrm{kHz}$, it is obtained that the $\varphi_{c}$ is $7.8 \mathrm{wt}$. $\%$ and the $\mathrm{s}$ is 1.9. From Figure 3(b), one can find that the fitting constant $\varphi_{c}$ can be as high as $8.3 \mathrm{wt} . \%$ and as low as $7.3 \mathrm{wt} . \%$, while the fitting constant s can be as high as 2.4 and as low as 0.8 . That is, if the dielectric constant at some higher frequencies is used, both the fitting constant $\varphi_{c}$ and $s$ would be reasonable.

The polarization mechanisms or relaxation processes in a composite is determined by the relaxation processes existing in each constituent and the new relaxation processes arising by the mixing of different materials, such as Maxwell-Wagner effect [5]. It should be mentioned that Eq. (1)/(4) was developed without considering the new relaxation process due to the mixing. Therefore, if the new relaxation process due to the mixing has a stronger contribution to the dielectric constant of a composite, Equation (1)/(4) may not be valid. As shown above, the relaxation frequency of the new relaxation process for the composites with 6 and 7 wt.\% PPy is above $1 \mathrm{kHz}$ so that the new relaxation process would have a stronger contribution to the dielectric constant at frequencies lower than $1 \mathrm{kHz}$. Regarding the fitting results, the dielectric constant of the composites with the $\varphi$ closer to the $\varphi_{c}$ has a much stronger influence on the fitting results. Therefore, for the composites reported here, the composites with 7 wt.\% PPy have the strongest, and the composites with 6 wt.\% PPy have the second strongest, influence on the fitting results/constant. Therefore, one can conclude that the new dielectric process has a stronger influence on the fitting results when the dielectric constant at frequencies lower than $1 \mathrm{kHz}$ is used. This may be the reason that the fitting constant $\varphi_{c}$ using Eq. (1)/(4) with the dielectric constant at low frequency is beyond its 
range. That is, when the new relaxation process has a strong contribution to the dielectric constant, Equation (1)/(4) may not be used.

The temperature dependence of the dielectric properties at $1 \mathrm{kHz}, 10 \mathrm{kHz}, 100 \mathrm{kHz}$, and $1 \mathrm{MHz}$ for all the composites is characterized over a temperature range from $-60{ }^{\circ} \mathrm{C}$ to $140{ }^{\circ} \mathrm{C}$. Some of the results are shown in Figure 4. To compare the dielectric properties of the composites with different PPy contents, the temperature dependence of both the dielectric constant and loss at $1 \mathrm{kHz}$ for all composites is plotted in Figure 5 (a) and (b). Clearly, the dielectric constant increases with increasing $\varphi$. For the composites with 7 wt.\% PPy, a dielectric constant at $100 \mathrm{~Hz}$ of more than 1,800 is obtained at temperatures above $98{ }^{\circ} \mathrm{C}$ with a loss as low as 2.2. For the composites with $8 \mathrm{wt} . \% \mathrm{PPy}$, a dielectric constant at $100 \mathrm{~Hz}$ of more than 2,000 is observed at temperatures higher than $98{ }^{\circ} \mathrm{C}$ with a loss as low as 2.7 . For other reported CDCs using 1-D fillers with such a high dielectric constant, their dielectric loss is usually much higher than the loss reported here [5].

For the pure matrix as shown in Figure 4 (a), there are two characteristic temperatures: 1) the glass-transition temperature $\left(T_{g}\right)$ which results in a typical relaxation process at temperatures ranging from $-20{ }^{\circ} \mathrm{C}$ to $+20{ }^{\circ} \mathrm{C}$ : a step-like increase on the dielectric constant and a loss peak; and 2) the ferroelectric-to-paraelectric phase transition temperature $\left(T_{F E}\right)$ around $100 \sim 110{ }^{\circ} \mathrm{C}$, which results in a maximum peak on both the dielectric constant and loss curve. For the relaxation process associated with $T_{g}$, both the loss-peak temperature and the loss at the peak increase with increasing frequency. At temperatures higher than $T_{F E}$, the loss at low frequency increases with the increasing temperature, which indicates that the polymer matrix at high temperature has a considerable electrical conductivity.

Regarding the influence of the glass-transition process of the matrix on the dielectric properties of the composites, the composites with the PPy content less than $5 \mathrm{wt} \%$ exhibit a typical relaxation process as evident by the loss peak and the step increase in the dielectric constant. However, some clear differences are observed. For example, for the composites with 3 wt.\% PPy, the difference in the loss-peak value among different frequencies is smaller than that observed in the matrix. Therefore, the composites exhibit a weaker temperature 
dependence of the dielectric constant at temperatures around $T_{g}$. For the composites with more than 5 wt.\% PPy, the loss peak associated with $T_{g}$ disappears. All these indicate that the appearance of PPy results in the glass-transition process of the matrix weaker or/and broader. This is exactly what was observed in the matrix of other composite systems [43].

Regarding the dielectric properties associated with the $T_{F E}$, it is found that, as the PPy content increases, the temperature dependence of the dielectric constant at temperatures around $T_{F E}$ becomes weaker, which results in a broader dielectric-constant peak at $T_{F E}$. Additionally, the $T_{F E}$ decreases slightly with increasing PPy content as shown in Figure 5 (a). That is, the appearance of PPy also has some influence on the phase transition process of the matrix. For the polymer matrix used here, it is known that the smaller the crystal size is, the lower the $T_{F E}$ is and that the lower the crystallinity is, the weaker the dielectric-constant peak associated with the $T_{F E}$ is [44]. Therefore, the results observed from the composites indicate that the appearance of PPy results in a lower crystallinity and smaller crystals in the matrix.

As shown in Figure 5 (b), the composites exhibit a loss higher than the matrix. To study the physics behind the high loss observed in the composites, the $\varepsilon_{r}{ }^{\prime \prime}$ is plotted in Figure 5 (c). Based on Equations. (2) and (3), one can get that the measured $\varepsilon_{r}{ }^{\prime \prime}$ is

$$
\varepsilon_{r}^{\prime \prime}=\varepsilon_{p}^{\prime \prime}+\frac{\sigma_{d c}}{\varepsilon_{0} \cdot \omega}
$$

The temperature dependence of the $\varepsilon_{p}$ " can be very complicated, but the temperature dependence of the conductivity can be simply written as [45]:

$$
\sigma_{d c}=\sigma_{0} \exp \left(-\frac{T_{a}}{T}\right)
$$

where $\sigma_{0}$ is the pre-exponential factor, $T_{a}$ is the equivalent temperature of the activation energy. First of all, Equation (6) indicates that the conductivity increases with increasing temperature. Therefore, the higher the temperature is, the stronger the contribution of the electrical conductivity to the measured $\varepsilon_{r}{ }^{\prime \prime}$. Equations (5) and (6) indicate that the $\varepsilon_{r}{ }^{\prime \prime}$ due to the conductivity should result in a linear relationship between the $\ln \left(\varepsilon_{r}{ }^{\prime \prime}\right)$ and $1 / \mathrm{T}$. Assuming the $\varepsilon_{r}{ }^{\prime \prime}$ at high temperature is dominated by the conductivity, a linear relationship is obtained 
for the $\varepsilon_{r}{ }^{\prime \prime}$ measured at $1 \mathrm{kHz}$ as shown in Figure 5 (c) as solid lines. The results indicate that the measured $\varepsilon_{r}$ " at room temperature is much higher than the contribution from the conductivity. For example, for composites with 7 wt.\% PPy, the conductivity only contributes to $9.8 \%$ of the measured $\varepsilon_{r}{ }^{\prime \prime}$. Even for the composites with $8 \mathrm{wt} . \%$ PPy, the conductivity's contribution to the measured $\varepsilon_{r}^{\prime \prime}$ at room temperature is only $20.0 \%$. Therefore, the loss observed in the composites at low temperature including room temperature is mainly determined by the dielectric relaxation process induced by the appearance of PPy.

Application Remarks: Soft electronics have attracted a great deal of attentions in recent years due to its applications in personal/wearable electronics, bioengineering, sensors and actuators, and energy storage devices [46]. The flexible dielectric composites with a high dielectric constant can be a great candidate for the fabrication of flexible capacitors and other dielectric devices which are critical for the soft electronics. All the composites reported here show a good flexibility. For example, a composite film with a length of less than $1.0 \mathrm{~cm}$ can be bended into more than one circle. For the flexible devices, a critical issue is their performance under different bending conditions or whether the performance changes with bending condition. For the composites studied here, their dielectric properties were characterized under different bending conditions. It is found that the dielectric loss of each and every composite does not change with the bending condition. However, it is found that the capacitance of the sample (i.e. composite film coated with circular gold electrodes on both surface) under bending condition is slightly higher than the capacitance of the flat sample. For example, for circular composite film with a diameter of about $1.1 \mathrm{~cm}$ coated with circular gold electrode with a diameter of $0.8 \mathrm{~cm}$, its capacitance of the sample bended to almost one circle is about 1.0 to $4.0 \%$ higher than the capacitance of the flat sample. It seems that the change in the capacitance caused by bending is independent of frequency. Additionally, no correlation between the change in the capacitance and the composition of the composites was obtained. More interestingly, it is found that the capacitance of the flat sample before and after bending is the same. All these indicate that the mechanical bending does not introduce any change in the composite. It is concluded: 1) the change in the 
capacitance caused by bending originates from the change in geometry of the samples; 2) the dielectric properties of the composites do not change with bending. The latter is necessary and important for the development of soft electronics. The reason for the former is that bending induces stresses in the composite film, which in turn results in strains in the film. That is, the bending would result in the change on the dimensions of the samples. Although the dielectric properties do not change with the bending, that is good for applications; the change in the capacitance due to the bending can become an issue for the soft electronics. Therefore, some detailed studies on this, such as fine element simulation, may be needed to be carried out to predict the change in device performance under different bending conditions. This should be an interesting topic for the soft electronics.

\section{Conclusions}

In conclusion, an all-organic composite system using newly developed PPy nanoclips is developed. The composites have a uniform microstructure due to the unique preparation process. The composites have a very low percolation threshold ( $<8 \mathrm{wt} . \%)$ and exhibit a high dielectric constant. At room temperature, the composites exhibit a dielectric constant of more than 1,000 . At temperatures higher than $98{ }^{\circ} \mathrm{C}$, the composites exhibit a dielectric constant of about 2,000. More interestingly, the high dielectric constant reported here is associated with a loss much smaller than the loss reported for other CDCs using 1-D fillers. It is indicated that a new dielectric relaxation process is induced due to the mixture of PPy with P(VDF-TrFE), whose relaxation time decreases with increasing PPy content. The loss observed in the composites at low temperature including room temperature is mainly determined by this relaxation process rather than the conductivity. If this relaxation process has a strong contribution to the dielectric constant, the widely used percolation formula describing relationship between the dielectric constant and the composite cannot be used.

\section{Acknowledgements}

This work at Auburn was supported by the USAD National Institute of Food and Agriculture, 
Nanotechnology for Agriculture and Food Systems (2014-67022-24925, 2015-07682), an AU-IGP grant, and a NASA grant (\#G00007275), while at Xi'an was supported by the "111" project (B14040). 


\section{References}

[1] Z. Cheng, Q. Zhang, MRS Bulletin, 33 (2008) 183-187.

[2] Z.-M. Dang, J.-K. Yuan, S.-H. Yao, R.-J. Liao, Advanced Materials, 25 (2013) 6334-6365.

[3] Z.-M. Dang, J.-K. Yuan, J.-W. Zha, T. Zhou, S.-T. Li, G.-H. Hu, Progress in Materials Science, 57 (2012) 660-723.

[4] C.W. Nan, Y. Shen, J. Ma, Annual Review of Materials Research, 40 (2010) 131-151.

[5] L.I.N. Zhang, Z.Y. Cheng, Journal of Advanced Dielectrics, 01 (2011) 389-406.

[6] Z.-M. Dang, Y. Zheng, H.-P. Xu, Journal of Applied Polymer Science, 110 (2008) 3473-3479.

[7] M.-a. Kakimoto, A. Takahashi, T.-a. Tsurumi, J. Hao, L. Li, R. Kikuchi, T. Miwa, T. Oono, S. Yamada, Materials Science and Engineering: B, 132 (2006) 74-78.

[8] P. Kim, S.C. Jones, P.J. Hotchkiss, J.N. Haddock, B. Kippelen, S.R. Marder, J.W. Perry, Advanced Materials, 19 (2007) 1001-1005.

[9] L. Zhang, P. Wu, Y. Li, Z.Y. Cheng, J.C. Brewer, Composites Part B: Engineering, 56 (2014) 284-289.

[10] H. Tang, H.A. Sodano, Nano Letters, 13 (2013) 1373-1379.

[11] Y. Bai, Z.-Y. Cheng, V. Bharti, H.S. Xu, Q.M. Zhang, Applied Physics Letters, 76 (2000) 3804-3806.

[12] M. Arbatti, X. Shan, Z.Y. Cheng, Advanced Materials, 19 (2007) 1369-1372.

[13] L. Zhang, X. Shan, P. Wu, Z.Y. Cheng, Applied Physics A, 107 (2012) 597-602.

[14] L. Zhang, X. Shan, P. Wu, J. Song, Z.Y. Cheng, Ferroelectrics, 405 (2010) 92-97.

[15] C. Ehrhardt, C. Fettkenhauer, J. Glenneberg, W. Munchgesang, H.S. Leipner, M.

Diestelhorst, S. Lemm, H. Beige, S.G. Ebbinghaus, Journal of Materials Chemistry A, 2 (2014) 2266-2274.

[16] H. Tang, Z. Zhou, C.C. Bowland, H.A. Sodano, Nano Energy, 17 (2015) 302-307.

[17] Z.M. Dang, Y.H. Lin, C.W. Nan, Advanced Materials, 15 (2003) 1625-1629.

[18] M. Panda, V. Srinivas, A.K. Thakur, Applied Physics Letters, 92 (2008) 132905.

[19] M. Panda, V. Srinivas, A.K. Thakur, Applied Physics Letters, 93 (2008) 242908.

[20] H.-P. Xu, H.-Q. Xie, D.-D. Yang, Y.-H. Wu, J.-R. Wang, Journal of Applied Polymer Science, 122 (2011) 3466-3473.

[21] L. Zhang, X. Wang, Z.Y. Cheng, Journal of Advanced Physics, 4 (2015) 362-369.

[22] L. Zhang, P. Bass, Z.-Y. Cheng, Applied Physics Letters, 105 (2014) 042905.

[23] L. Zhang, W. Wang, X. Wang, P. Bass, Z.-Y. Cheng, Applied Physics Letters, 103 (2013) 232903.

[24] Z.M. Dang, L. Wang, Y. Yin, Q. Zhang, Q.Q. Lei, Advanced Materials, 19 (2007) 852-857.

[25] Q. Li, Q. Xue, L. Hao, X. Gao, Q. Zheng, Composites Science and Technology, 68 (2008) 2290-2296.

[26] W. Xu, Y. Ding, S. Jiang, J. Zhu, W. Ye, Y. Shen, H. Hou, European Polymer Journal, 59 (2014) 129-135. 
[27] W. Xu, Y. Feng, Y. Ding, S. Jiang, H. Fang, H. Hou, Materials Letters, 161 (2015) 431-434.

[28] J.-K. Yuan, S.-H. Yao, Z.-M. Dang, A. Sylvestre, M. Genestoux, J. Bai, The Journal of Physical Chemistry C, 115 (2011) 5515-5521.

[29] F. He, S. Lau, H.L. Chan, J. Fan, Advanced Materials, 21 (2009) 710-715.

[30] V. Panwar, R.M. Mehra, J.-O. Park, S.-h. Park, Journal of Applied Polymer Science, 125 (2012) E610-E619.

[31] Q.M. Zhang, H. Li, M. Poh, F. Xia, Z.Y. Cheng, H. Xu, C. Huang, Nature, 419 (2002) 284-287.

[32] C. Huang, Q. Zhang, Advanced Functional Materials, 14 (2004) 501-506.

[33] C.-C. Wang, J.-F. Song, H.-M. Bao, Q.-D. Shen, C.-Z. Yang, Advanced Functional Materials, 18 (2008) 1299-1306.

[34] J.-K. Yuan, Z.-M. Dang, S.-H. Yao, J.-W. Zha, T. Zhou, S.-T. Li, J. Bai, Journal of Materials Chemistry, 20 (2010) 2441-2447.

[35] K. Shehzad, A. Ul-Haq, S. Ahmad, M. Mumtaz, T. Hussain, A. Mujahid, A. Shah, M. Choudhry, I. Khokhar, S. Ul-Hassan, F. Nawaz, F. Rahman, Y. Butt, M. Pervaiz, Journal of Materials Science, 48 (2013) 3737-3744.

[36] Z. Liu, X. Zhang, S. Poyraz, S.P. Surwade, S.K. Manohar, Journal of the American Chemical Society, 132 (2010) 13158-13159.

[37] X. Zhang, Z. Liu, Nanoscale, 4 (2012) 707-714.

[38] X. Zhang, S.K. Manohar, Journal of the American Chemical Society, 126 (2004) 12714-12715.

[39] Z. Liu, Y. Liu, S. Poyraz, X. Zhang, Chemical Communications, 47 (2011) 4421-4423.

[40] M.T. Clarkson, Physical Review A, 37 (1988) 2079-2090.

[41] J.-K. Yuan, Z.-M. Dang, J. Bai, physica status solidi (RRL) - Rapid Research Letters, 2 (2008) 233-235.

[42] J. Macutkevic, D. Seliuta, G. Valušis, J. Banys, V. Kuznetsov, S. Moseenkov, O. Shenderova, Applied Physics Letters, 95 (2009) 112901.

[43] A. Bansal, H. Yang, C. Li, K. Cho, B.C. Benicewicz, S.K. Kumar, L.S. Schadler, Nat Mater, 4 (2005) 693-698.

[44] Z.-Y. Cheng, Q.M. Zhang, F.B. Bateman, Journal of Applied Physics, 92 (2002) 6749-6755.

[45] R.E. Hummel, Electronic Properties of Materials, Springer, New York, 2001.

[46] D.-H. Kim, R. Ghaffari, N. Lu, J.A. Rogers, Annual Review of Biomedical Engineering, 14 (2012) 113-128. 


\section{Figures captions:}

Figure 1. SEM images: (a) and (b) PPy nanoclips, (c) cross-section of one layer as-cast composite, (d) top surface of one layer composite, (e) and (f) cross-section of 4-layer hot-pressed composite with 5 wt.\% PPy nanoclips.

Figure 2. Frequency dependence of (a) dielectric constant, (b) loss, and (c) ac conductivity of PPy-P(VDF-TrFE) composites at room temperature.

Figure 3. (a) $\varepsilon_{r} / \varepsilon_{m}$ at $1 \mathrm{kHz}$ versus the composition $(\varphi)$ for PPy-P(VDF-TrFE) composites. Inset figure shows the fitting curve using Eq.(4). (b) The fitting constant $\varphi_{c}$ and $s$ of Eq.(4) using dielectric constant at different frequencies versus the frequency used.

Figure 4. Temperature dependence of dielectric constant (solid) and loss (open) of PPy-P(VDF-TrFE) with different contents of PPy nanoclips: (a) 0 wt.\%, (b) 3 wt.\%, (c) 5 wt.\%, (d) 7 wt.\% (e) 8 wt. \%, and (f) 9 wt.\%.

Figure 5. (a) Dielectric constant and (b) loss versus temperature (T) for the composites with different contents of PPy, (c) imaginary part of dielectric permittivity at $1 \mathrm{kHz}$ versus 1000/T for composites with 6,7 , and $8 \mathrm{wt} . \%$ to $8 \mathrm{wt} . \%$, where the solid lines are the fitting curves using the data at high temperature. 
Figure 1

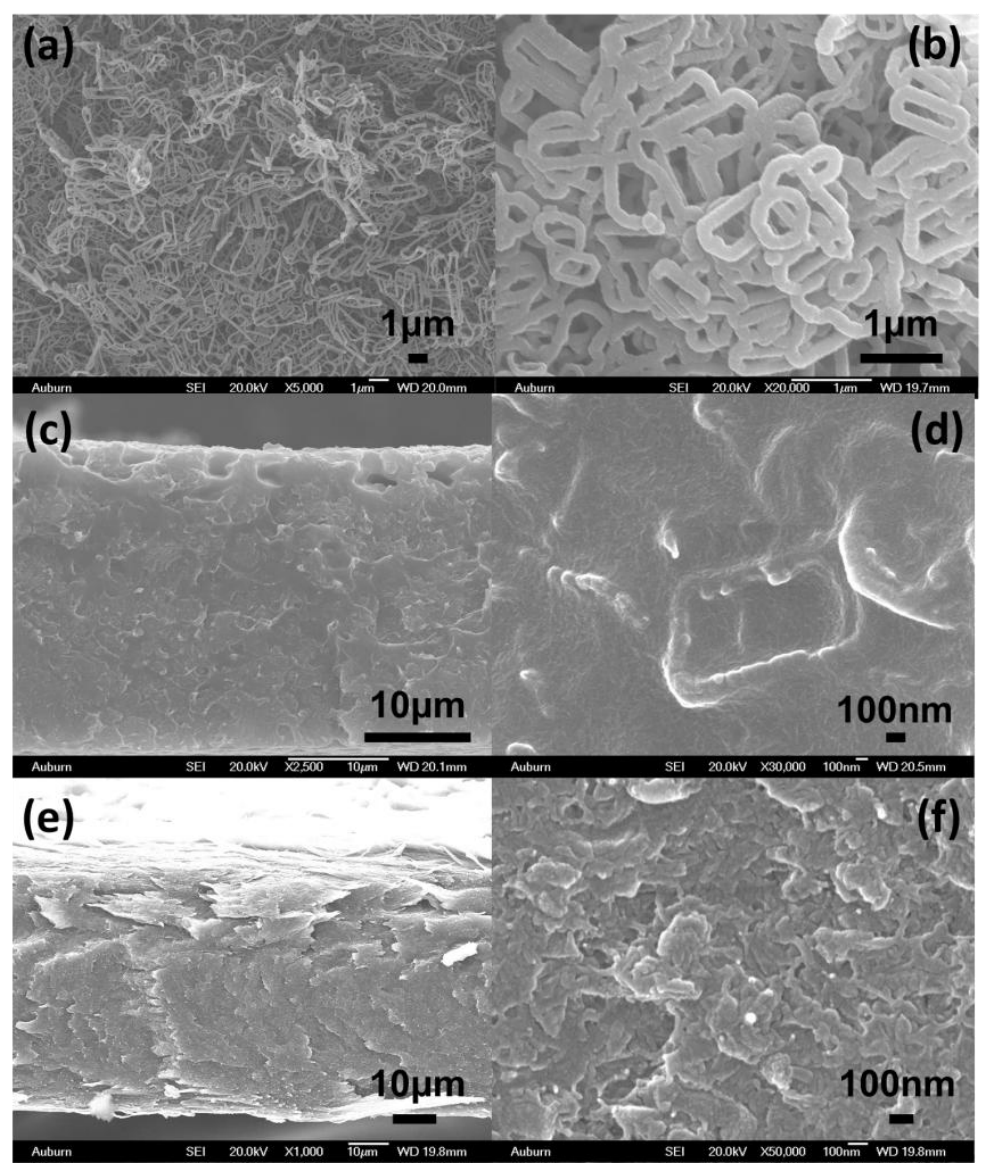


Figure 2
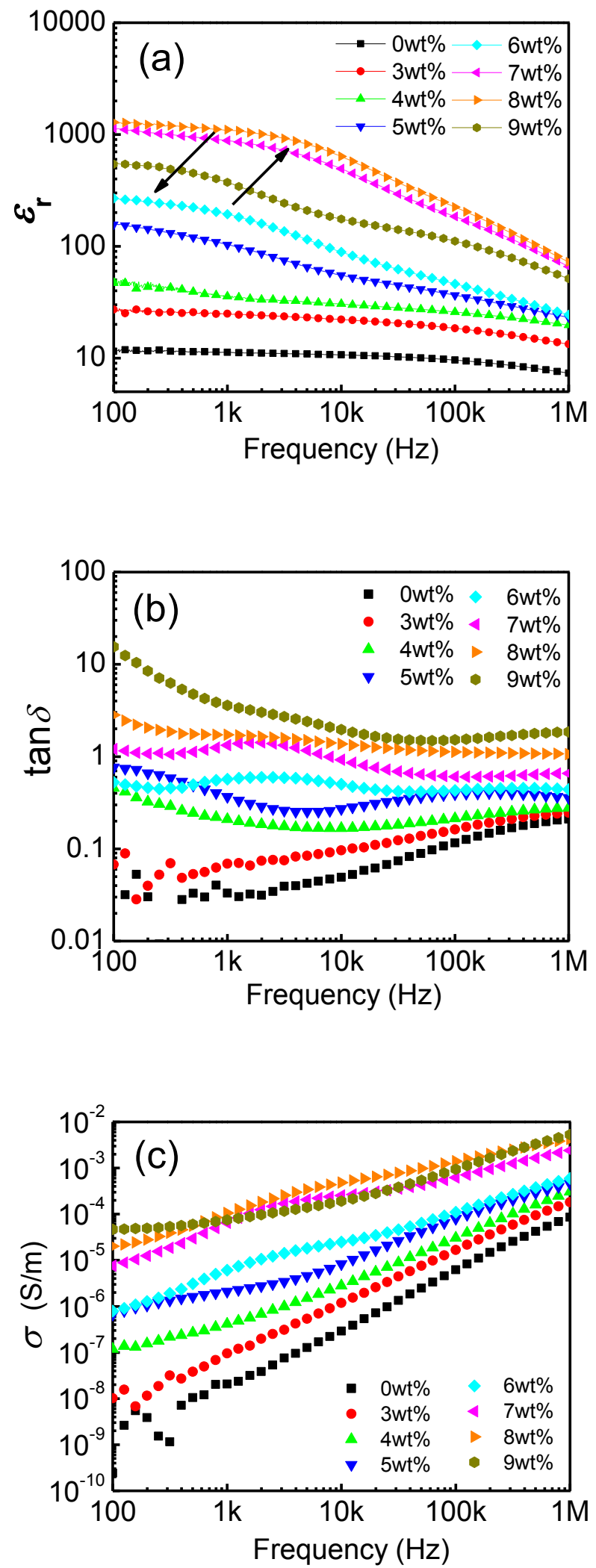
Figure 3
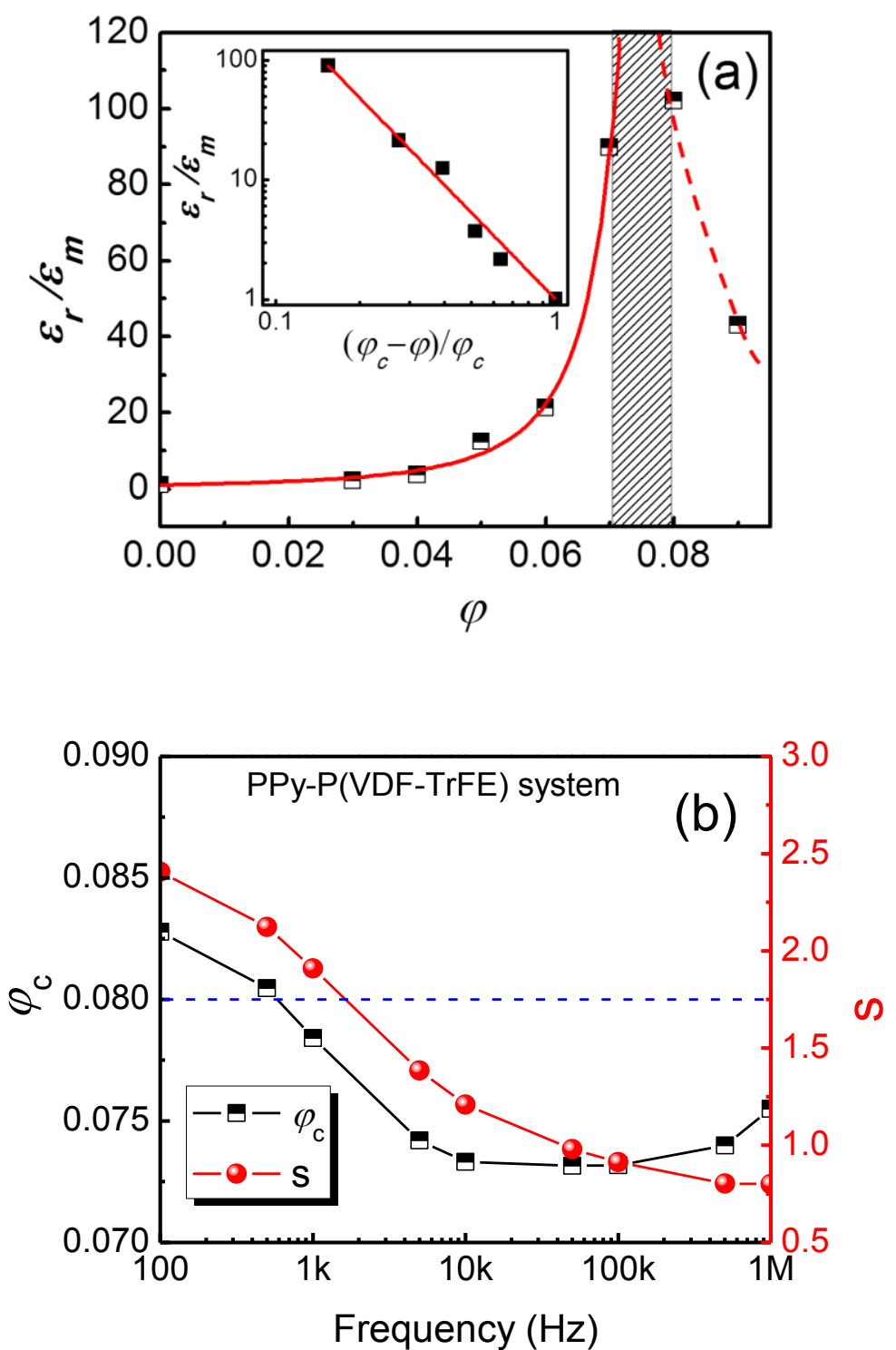
Figure 4
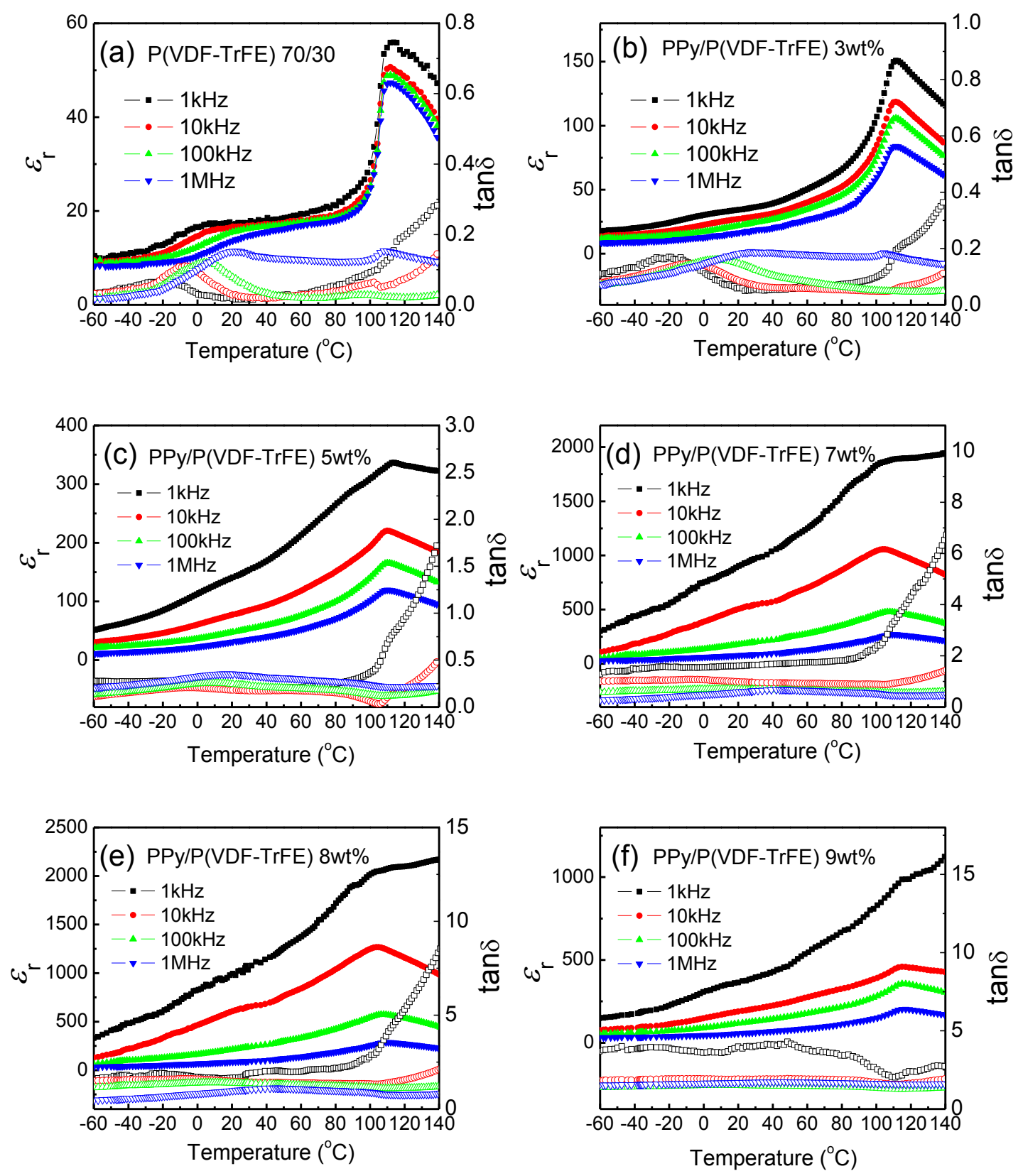
Figure 5
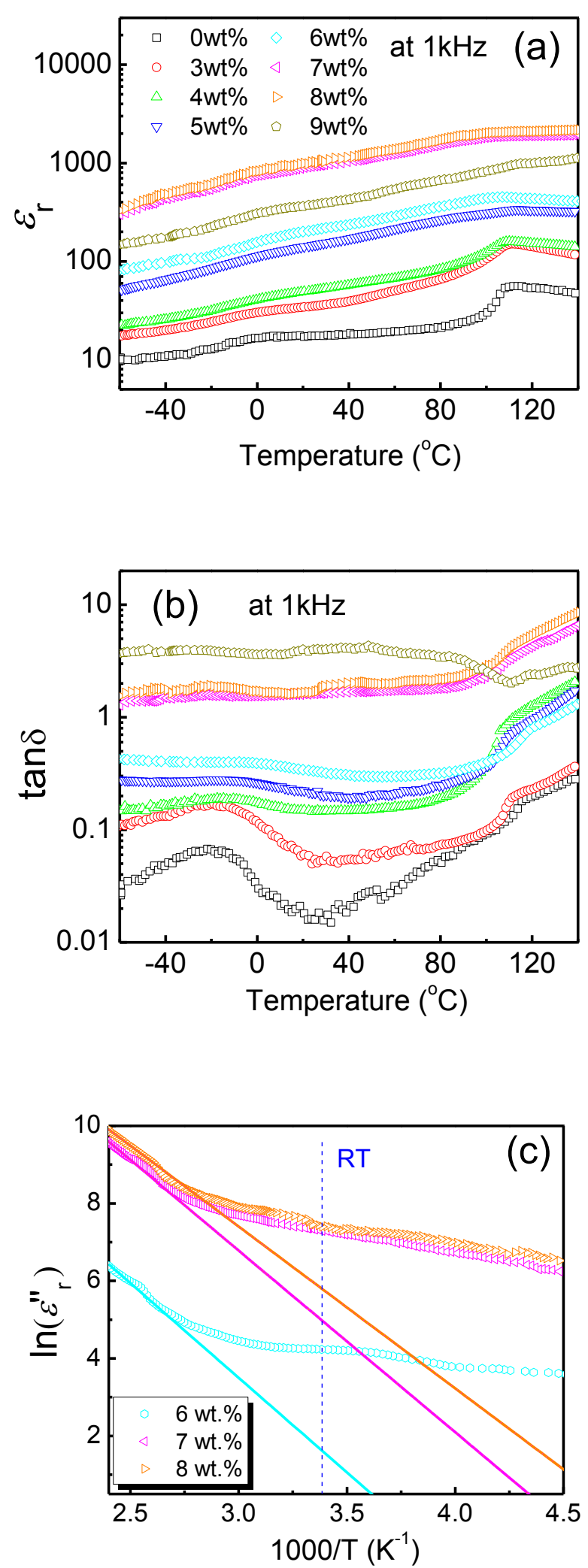


\section{Personal portrait photo and the biosketch}

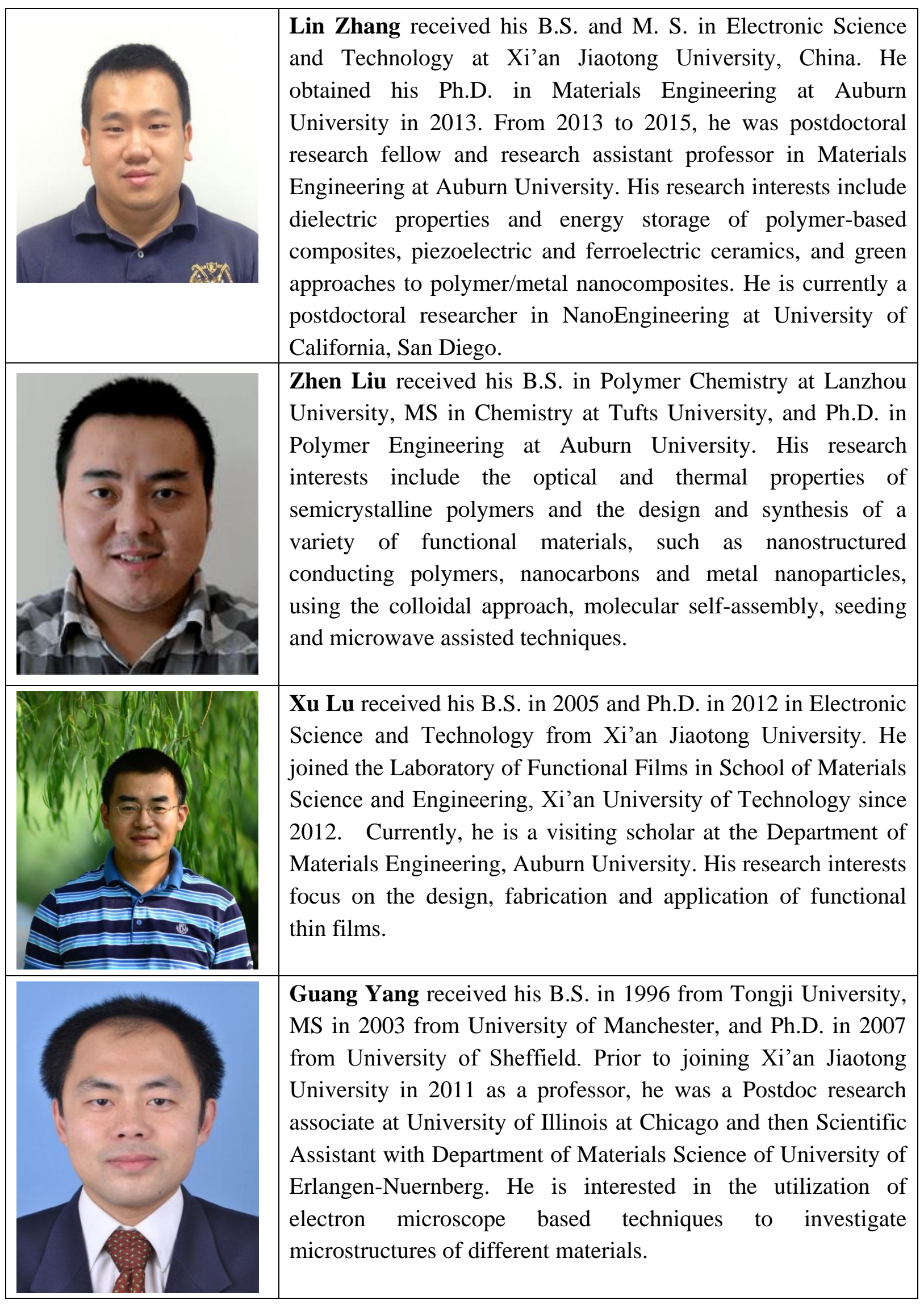




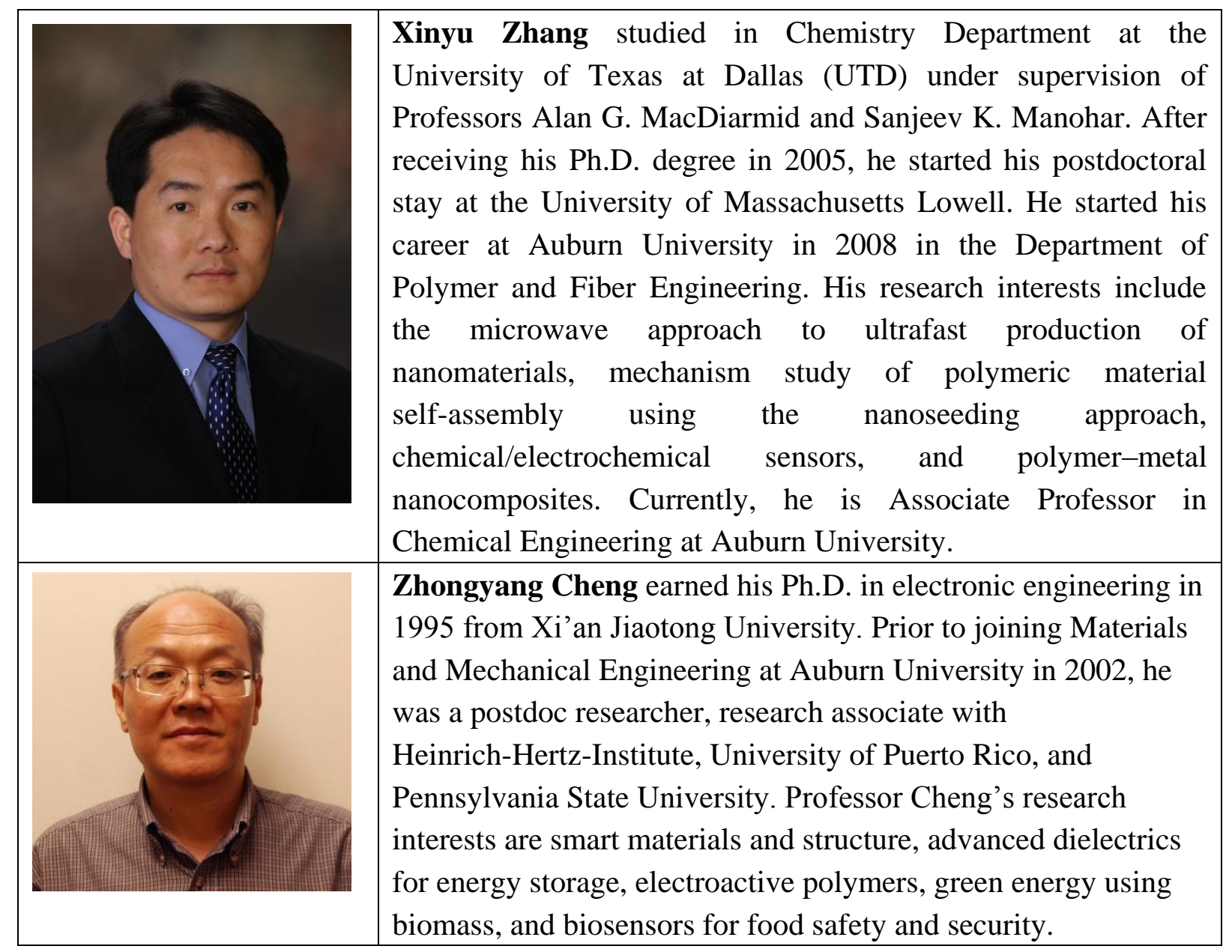

